\title{
INFLUENCE OF VARIOUS LIGHT TIMBER RETROFIT LAYOUTS ON THE DYNAMIC RESPONSE OF TYPICAL DUTCH MASONRY TERRACED HOUSES
}

\author{
C. MORANDINI ${ }^{1 *}$, D. MALOMO ${ }^{2}$, R. PINHO ${ }^{1,2}$ AND A. PENNA ${ }^{1}$ \\ ${ }^{1}$ University of Pavia, Via Ferrata 3, 27100, Pavia, Italy \\ e-mail: chiara.morandini02@universitadipavia.it*, andrea.penna@unipv.it (*corresponding author) \\ ${ }^{2}$ Mosayk Ltd. Via Fratelli Cuzio, 42, 27100, Pavia, Italy \\ email: daniele.malomo@mosayk.it, rui.pinho@mosayk.it
}

Keywords: timber retrofit, masonry; numerical modelling; Applied Element Method; shaketable test

\begin{abstract}
A majority of the residential building stock in Groningen (The Netherlands), which has been lately exposed to low intensity ground motions due to gas extraction, consists of unreinforced masonry (URM) structures not originally designed to withstand earthquakes. Amongst them, the terraced house building typology proved to be particularly vulnerable towards horizontal actions. Experimental results from a recently performed shake-table test on a full-scale terraced house prototype, characterised by the presence of a timber retrofitting system, seem to indicate that the dynamic response of these structures might be consistently improved through the employment of such a cost-effective light retrofitting solution. In this work, an advanced discontinuum-based model, implemented in the framework of the Applied Element Method (AEM), is developed to extend experimental results and to numerically investigate the influence of a number of additional timber retrofit layouts, characterised by different geometrical configurations, on the building behaviour. Each timber component was explicitly represented in the AEM models to represent the possible interaction among URM walls and the retrofitting system. First, the proposed modelling strategy is validated against experimental tests on both non-retrofitted and retrofitted URM panels subjected to cyclic shearcompression loading. Then, calibrated mechanical parameters were directly implemented in the full-scale building model. Given the good agreement between numerical and experimental outcomes in terms of both damage evolution and hysteretic response, a comprehensive parametric study was undertaken. Numerical evidence seems to suggest that the employment of different retrofit layouts may have a significant influence on the dynamic behaviour of the selected building typology.
\end{abstract}

\section{INTRODUCTION}

The Applied Element Method, originally conceived by Meguro and Tagel-Din [1] for simulating controlled demolition processes of both reinforced concrete (RC) and steel structures, is emerging as a reliable computational approach for the collapse simulation of large 
scale unreinforced masonry (URM) systems, as witnessed by various recent applications (e.g.[2,3]). In this work, AEM-based models are used to numerically investigate the impact of various timber retrofit layouts on the dynamic response of unreinforced masonry (URM) fullscale building specimens, representative of an end unit of a series of adjacent terraced house constructions typically found in the Groningen region, now exposed to induced seismicity [4]. The models are first validated against the results of in-plane tests on both isolated retrofitted and non-retrofitted masonry piers, as well as shake-table tests on a full-scale timber-retrofitted building specimen [5], carried out at the EUCENTRE laboratory (Pavia, Italy) in the context of a wider experimental campaign [6]. As depicted in Figure 1, the considered building specimen, (hereinafter referred to as EUC-BUILD7) is a two-storey URM building with an RC slab at the first floor, a timber diaphragm at the second floor and a timber roof. The walls are made of two leaves: an inner loadbearing calcium-silicate (CS) brick leaf and an outer clay (CL) brick leaf with only aesthetic and insulating functions). Both walls were constructed with a periodic (running-bond) arrangement and were connected to each other by steel ties. As the specimen represents an end unit, the south façade consists of a single CS leaf. The retrofitting system, introduced to improve the seismic performance, includes a timber frame (strong-backs and nogging elements) and Oriented Strand Board (OSB) panels connected to the longitudinal CS walls by screwed steel angles The experimentally-employed retrofitting scheme also included the stiffening of the second timber floor through the application of OSB panels and the reinforcement of North and South façades with vertical timber elements, connected to CS walls

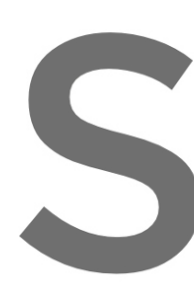
by screwed steel angl. reader is referred to Miglietta
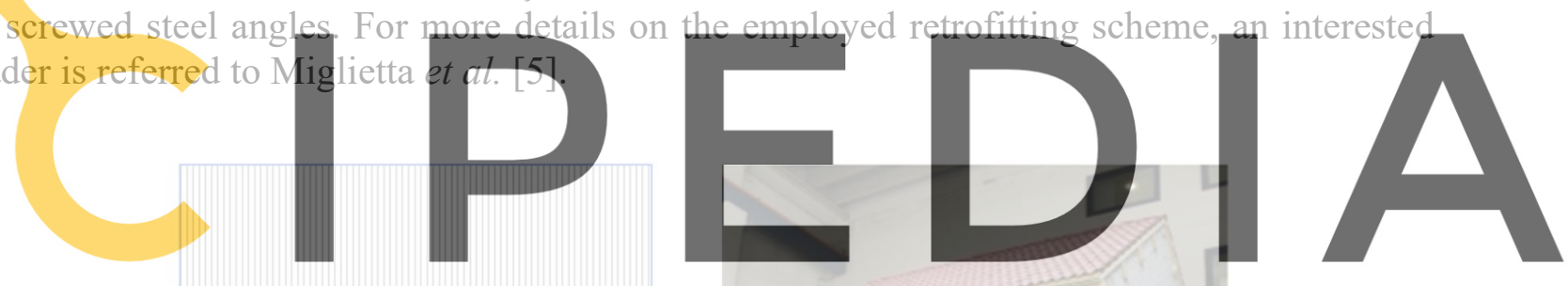

Register for free at https//wWw.Scipedia.com to download the version without the watermark

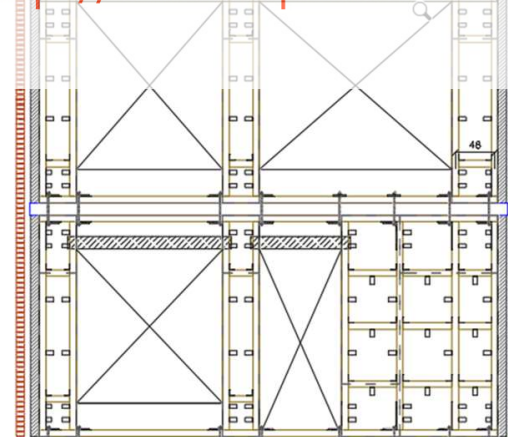

a

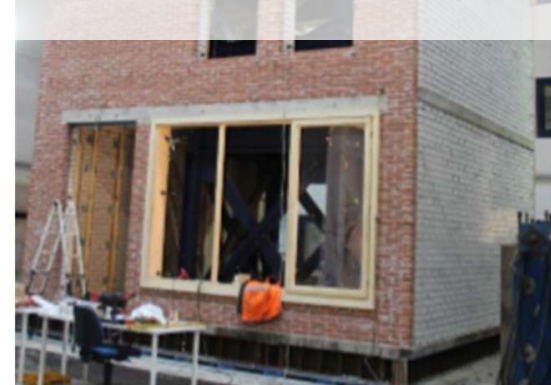

$\mathrm{b}$

Figure 1 a) East CS wall (OSB not represented, a) building specimen before testing [7] 


\section{NUMERICAL MODEL}

According to AEM, a given URM structure is idealised as an assembly of rigid units connected by linear and nonlinear springs, where the mechanical properties of the system are lumped. The main characteristics of the proposed AEM-based modelling strategy are summarised below (Figure 2) while interested readers may refer to $[8,9,10]$ for further details:

- A brick-based discretisation is used to reproduce the real arrangement of masonry. Fully rigid units are employed, thus neglecting the development of cracks within the bricks.

- First floor RC slab is idealised as a rigid diaphragm. Second strengthened floor and roof are modelled as elasto-plastic membranes, calibrated through comparison with experimental tests on components [11].

- Tie-connections between CS and CL walls are idealised as bilinear beam elements.

- As is typically done in common construction practice for this type of buildings, the gap that remained between the bottom surface of the RC floor slabs and the top edge of the CS longitudinal façades was filled with mortar only after the attainment of gravity loads, which essentially acted only on the transversal CS walls. The abovementioned construction process has been faithfully reproduced numerically and, since flexural and shear stiffness were likely to be limited due to lack of vertical compression, reduced values have been assigned to the corresponding interface springs.
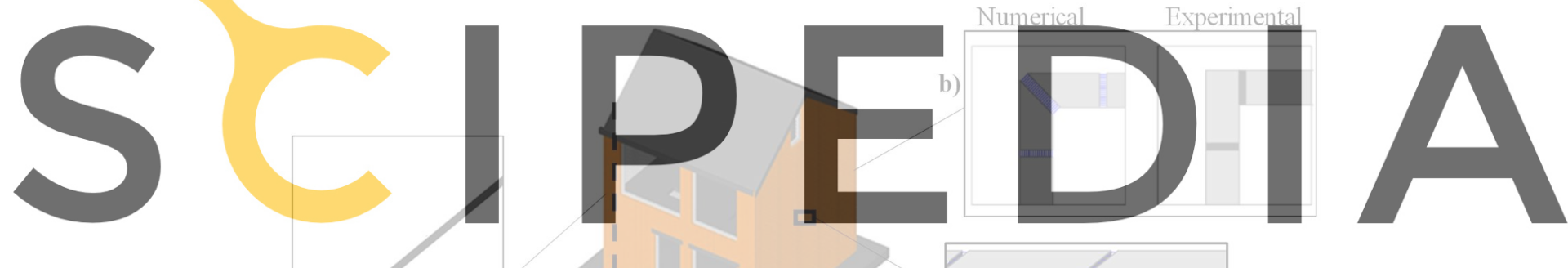

Register for free at https//www'scipedia.com to download the version without the watermark
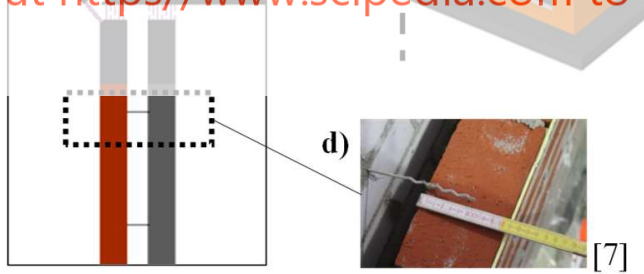

d)
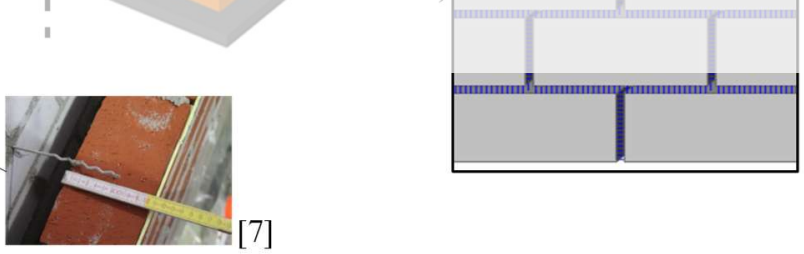

Figure 2 AEM discretisation of a 3D masonry segment (a), construction details and their numerical counterparts (b,c,d), (adapted from [12])

In addition to the abovementioned, other features related to the modelling of the timber retrofit system (see [12]) are also of relevance (Figure 3):

- Strong-backs (in green) and nogging elements (in yellow) are modelled as beam elements, with cross-section comparable to their experimental counterparts, and characterised by a symmetric elasto-plastic behaviour. The deformability of the strongbacks and nogging elements assembly is provided by the introduction of an equivalent yield stress, which has been iteratively calibrated simulating the in-plane response of 
the retrofitted wall specimen tested under cyclic shear-compression loading (see section $3)$.

- Strong-backs and nogging elements are rigidly connected to each other through interface 1.

- Since cracks only affect masonry and no damage was experimentally detected on steel angles connecting timber to masonry elements, in the model the connection between strong-backs/nogging elements and the small cuboids (in light blue), fixed to the CS walls through interface 2 , is assumed to be rigid. The retrofitting system deformability is thus lumped into interfaces 4 and 6.

- OSB panels (in light red) are modelled as bi-linear equivalent membranes and they are connected, through spring interface 3 , to both strong-backs and nogging elements.

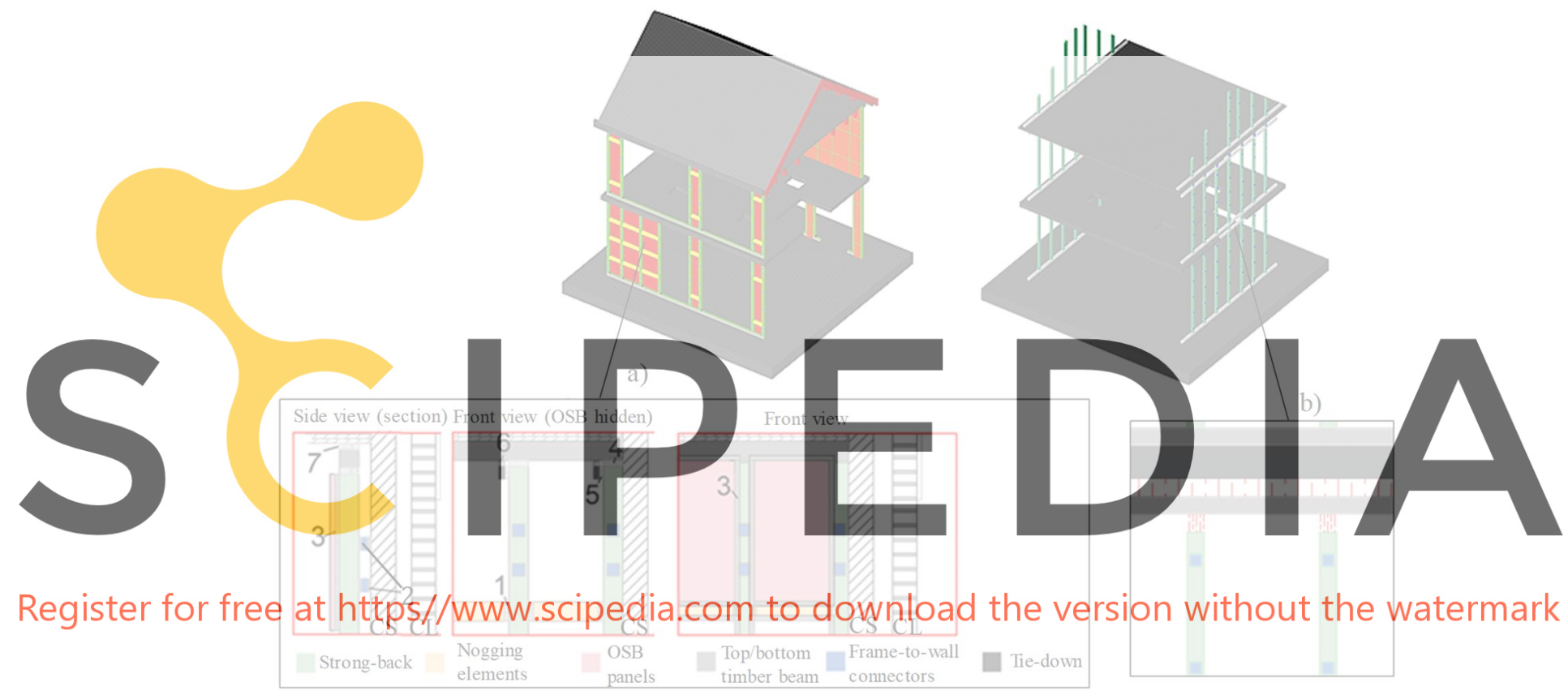

Figure 3 Construction details of the retrofit solution and numerical idealisations [12]

- The strong-backs are connected to the top/bottom timber beams through spring interface $\underline{4}$, to which zero tensile strength and zero cohesion has been allotted (i.e. dry joints)

- Tie-down connectors (in dark grey) are represented by rigid elements fixed to the strong-backs (interface 5). Their deformability is lumped into spring interface 6, characterised by an elasto-plastic behaviour and by an equivalent Young's modulus introduced to consider the buckling failure exhibited during the in-plane tests of EUCCOMP2 [5].

- The whole timber frame is fixed to both bottom and top slabs or floor diaphragm through interface 7.

As the in-plane (IP) one, the out-of-plane (OOP) retrofit was explicitly modelled [12]:

- Strong-backs, wall-to-strong-backs connection elements and interfaces properties are equal to the IP ones. 
- Strong-back elements are fixed to the top/bottom timber beams which are fixed to the foundation, to the $1^{\text {st }}$ floor or $2^{\text {nd }}$ floor.

In Table 1, the main material properties, obtained through characterisation tests, analytical expressions and calibration process, as discussed in e.g. [8], are reported. These include: compressive strength, $f_{c m}$ and Young's modulus, $E_{m}$, of masonry, flexural bond strength $\mathrm{f}_{\mathrm{w}}$, cohesion, c, friction coefficient, $\mu$, and Young's modulus, $E_{m o}$, of mortar. $E_{w}, G_{w}, f_{w}$ and $E_{O S B}$, $G_{O S B}, f_{y}, O S B$ are the numerical value for Young's modulus, shear modulus and yield stress assumed for wood and OSB panels respectively.

Table 1: Experimental and inferred masonry and retrofitting system mechanical properties

\begin{tabular}{|c|c|c|c|c|c|c|c|c|c|c|c|}
\hline \multicolumn{6}{|c|}{$\mathrm{CS}-$ density $\rho_{m}=1862[\mathrm{~kg} / \mathrm{m} 3]$} & \multicolumn{6}{|c|}{$\mathrm{CL}-$ density $\rho_{m}=12072[\mathrm{~kg} / \mathrm{m} 3]$} \\
\hline $\begin{array}{c}f c_{m} \\
{[\mathrm{MPa}]}\end{array}$ & $\begin{array}{c}f_{w} \\
{[\mathrm{MPa}]}\end{array}$ & $\begin{array}{c}E_{m} \\
{[\mathrm{MPa}]}\end{array}$ & $\begin{array}{c}c \\
{[\mathrm{MPa}]}\end{array}$ & $\begin{array}{l}\mu \\
{[-]}\end{array}$ & $\begin{array}{c}E_{m o} \\
{[\mathrm{MPa}]}\end{array}$ & $\begin{array}{c}f_{c m} \\
{[\mathrm{MPa}]}\end{array}$ & $\begin{array}{c}f_{w} \\
{[\mathrm{MPa}]}\end{array}$ & $\begin{array}{c}E_{m} \\
{[\mathrm{MPa}]}\end{array}$ & $\begin{array}{c}c \\
{[\mathrm{MPa}]}\end{array}$ & $\begin{array}{l}\mu \\
{[-]}\end{array}$ & $\begin{array}{c}E_{m o} \\
{[\mathrm{MPa}]}\end{array}$ \\
\hline 10.05 & 0.33 & 7319 & 0.62 & 0.71 & 6593 & 17.62 & 0.14 & 5686 & 0.3 & 0.62 & 1104 \\
\hline \multicolumn{6}{|c|}{ Timber - density $\rho=517[\mathrm{~kg} / \mathrm{m} 3]$} & \multicolumn{6}{|c|}{ OSB - density $\rho=572[\mathrm{~kg} / \mathrm{m} 3]$} \\
\hline \multicolumn{2}{|c|}{$E_{w}[\mathrm{MPa}]$} & \multicolumn{2}{|c|}{$G_{w}[\mathrm{MPa}]$} & \multicolumn{2}{|c|}{$f_{y}[\mathrm{MPa}]$} & \multirow{2}{*}{\multicolumn{2}{|c|}{$\frac{E_{O S B}[\mathrm{MPa}]}{1200}$}} & \multirow{2}{*}{\multicolumn{3}{|c|}{$\frac{G_{O S B}[\mathrm{MPa}]}{500}$}} & $f_{y}[\mathrm{MPa}]$ \\
\hline & & & 0 & & 10 & & & & & & 9 \\
\hline
\end{tabular}

\section{SIMULATION OF COMPONENT TESTS}

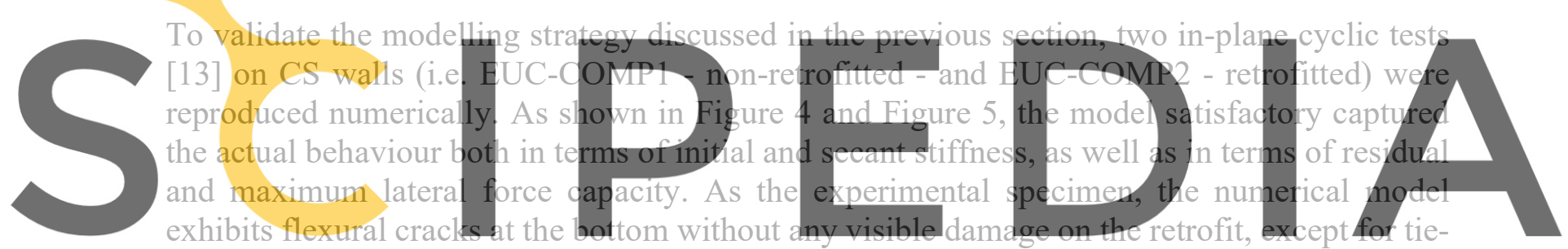

down connections, which suffered buckling-induced failures.

Register for free at https//www.scipedia.com to download the version without the watermark

a)

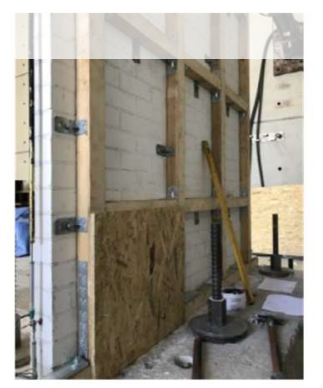

b)

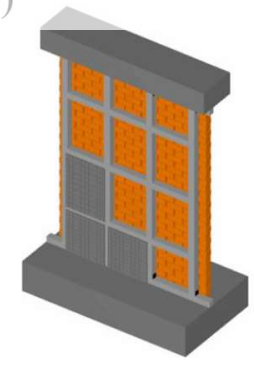

c)

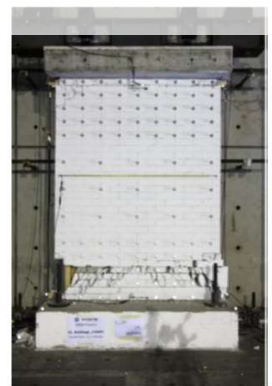

d)

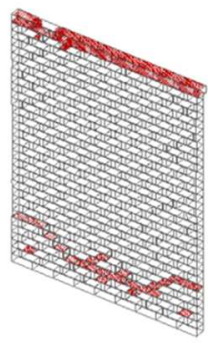

Figure 4 Experimental specimen a), numerical model b), experimental and numerical damage pattern c,d) of EUC-COMP2 [12,13] 


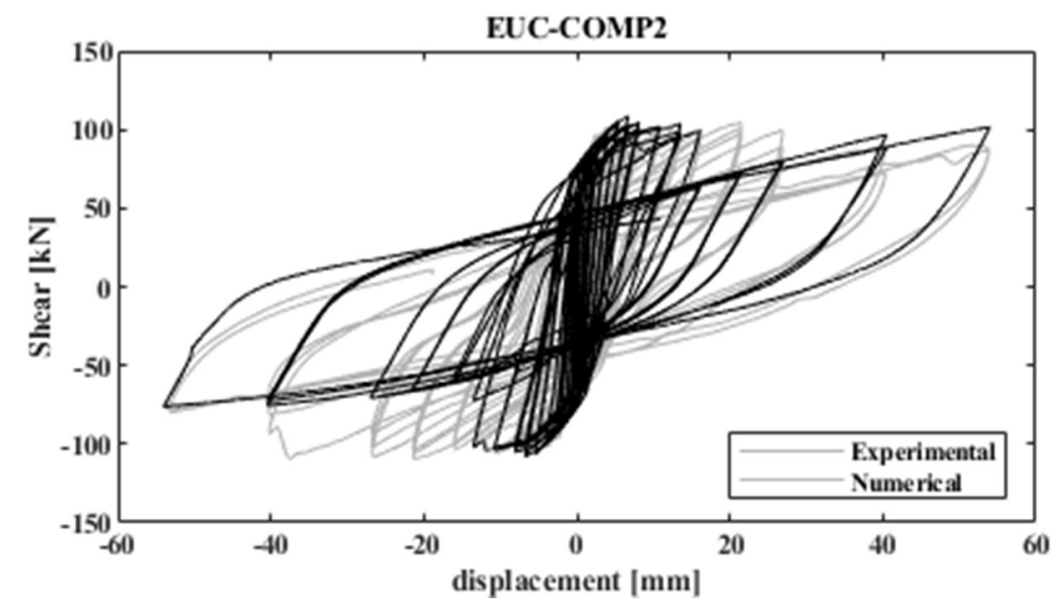

Figure 5 Numerical and experimental hysteretic response of EUC-COMP2 $[12,13]$

\section{SIMULATION OF THE FULL-SCALE SHAKE TABLE TEST}

The building specimen EUC-BUILD7 was subjected to a series of incremental dynamic inputs until up to near collapse conditions, as discussed in [5,7]. The numerical model behaviour, mainly governed by the first storey displacement demand, adequately reproduced the experimental response, both in terms of first and second floor displacements/inter-storey drifts (evaluated as first floor displacement divided by first-storey height or difference between second and first floor displacements divided by both URM leaves and residual and maximum

Figure 7. In the experiment, the damage cracks in both longitudinal and transversal significant difference on stiffiness between E

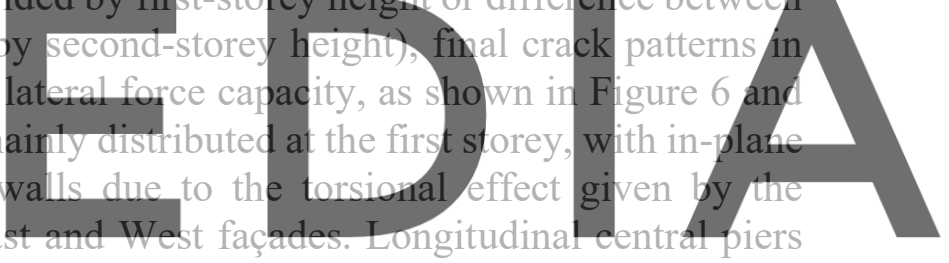
generally exhibited flexural/rocking behaviour in both the numerical model and the specimen,

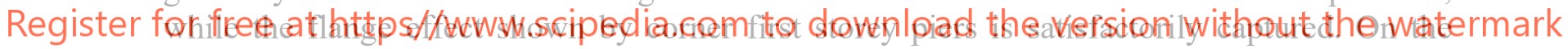
contrary, transversal walls damage has been slightly underestimated by the model, most likely because of the simplified assumption made for the corner connection between orthogonal walls.

a)

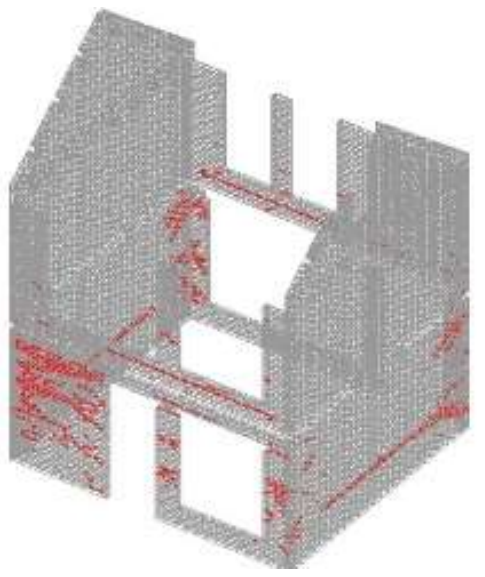

b)

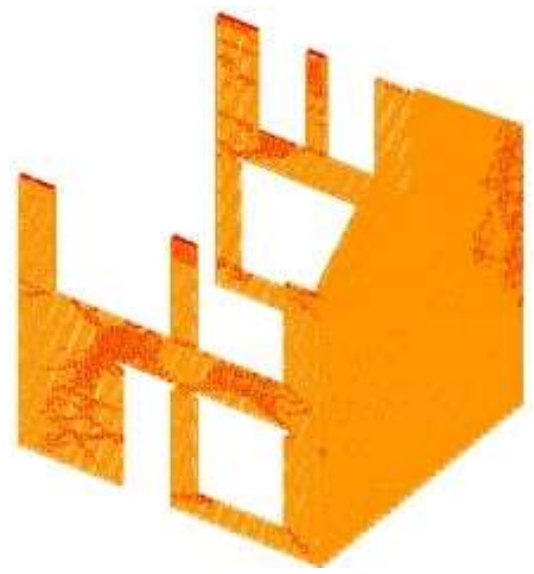

Figure 6 Numerical crack patterns of both CS and CL leaves a,b) [12] 

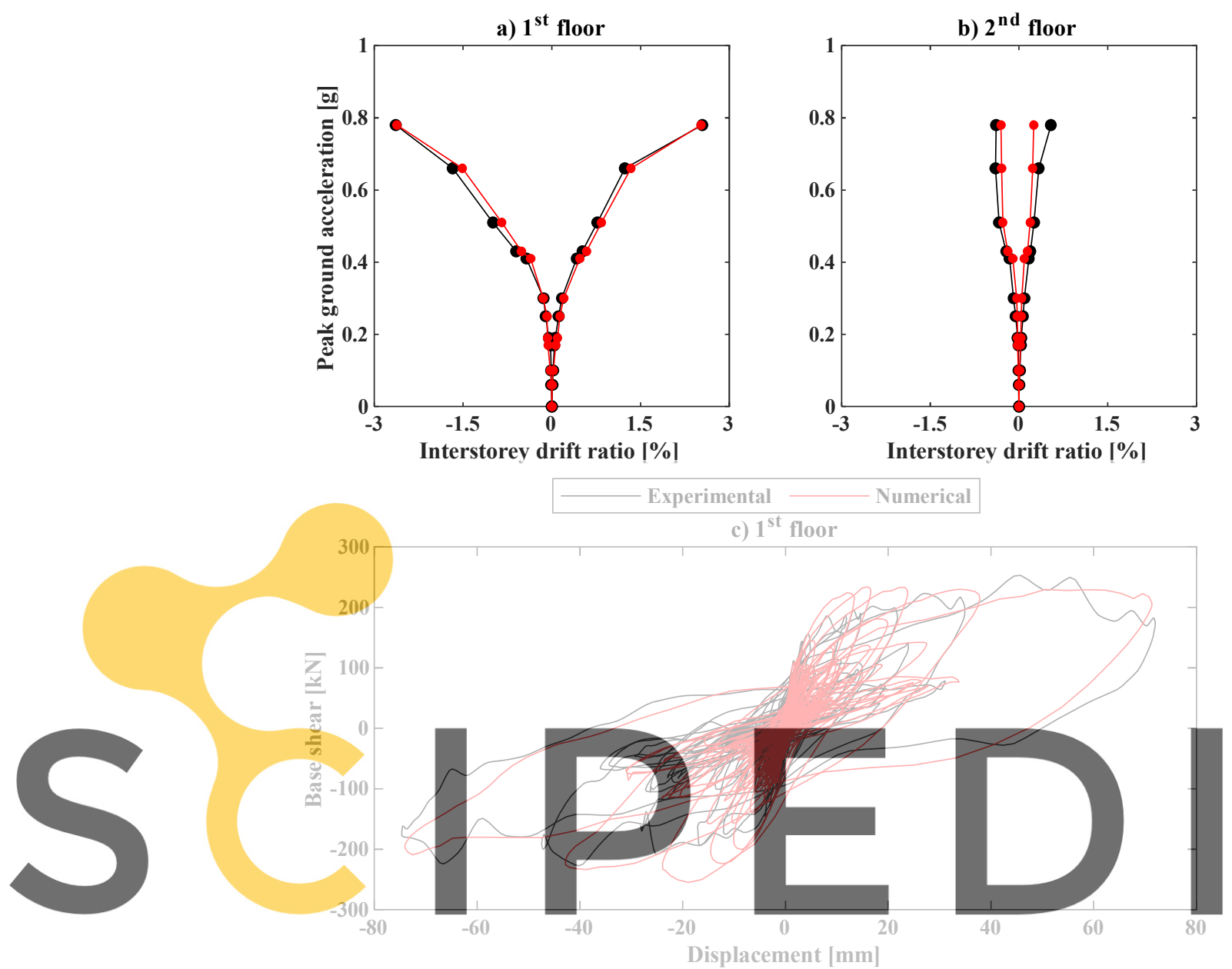

Register for free at https//www.scipedia.com to download the version without the watermark

Figure 7 Experimental vs Numerical comparison in terms of global hysteretic curves c) and IDA a,b) [12]

\section{PARAMETRIC ANALYSIS}

In this section, a brief overview of the parametric analysis, performed to investigate the impact of different additional retrofit layouts, is presented. Starting from the baseline model (i.e. EUC-BUILD7), four different configurations are generated varying horizontal and vertical timber frame span (i.e. LH and LV) and element dimensions (i.e. D1 and D2). The same dynamic loading protocol experimentally employed for EUC-BUILD7 was considered. In more details, the following retrofit configurations, reported in Figure 8, are considered:

- Configuration D1 with the same geometry of the baseline retrofitting system and strong-backs/nogging elements section reduced from $80 \mathrm{~mm}$ x $60 \mathrm{~mm}$ to $80 \mathrm{~mm}$ x $40 \mathrm{~mm}$ (where the reduced dimension is the one perpendicular to the masonry wall), (Figure 8 a,d). 
- Configuration D2 with the same geometry of the baseline retrofitting system and strong-backs/nogging elements section increased from $80 \mathrm{~mm}$ x $60 \mathrm{~mm}$ to $80 \mathrm{~mm}$ x $80 \mathrm{~mm}$ (Figure 8 a,d).

- Configuration LH with strong-backs/nogging elements cross-section equal to the one of baseline retrofitting system (i.e. $80 \mathrm{~mm}$ x $60 \mathrm{~mm}$ ), (Figure 8 a,b), with horizontal frame span reduced with respect to the original layout.

- Configuration LV with strong-backs/nogging elements cross-section equal to the one of baseline retrofitting system (i.e. $80 \mathrm{~mm}$ x $60 \mathrm{~mm}$ ), (Figure $8 \mathrm{a}, \mathrm{c}$ ), with vertical frame span reduced with respect to the original layout.

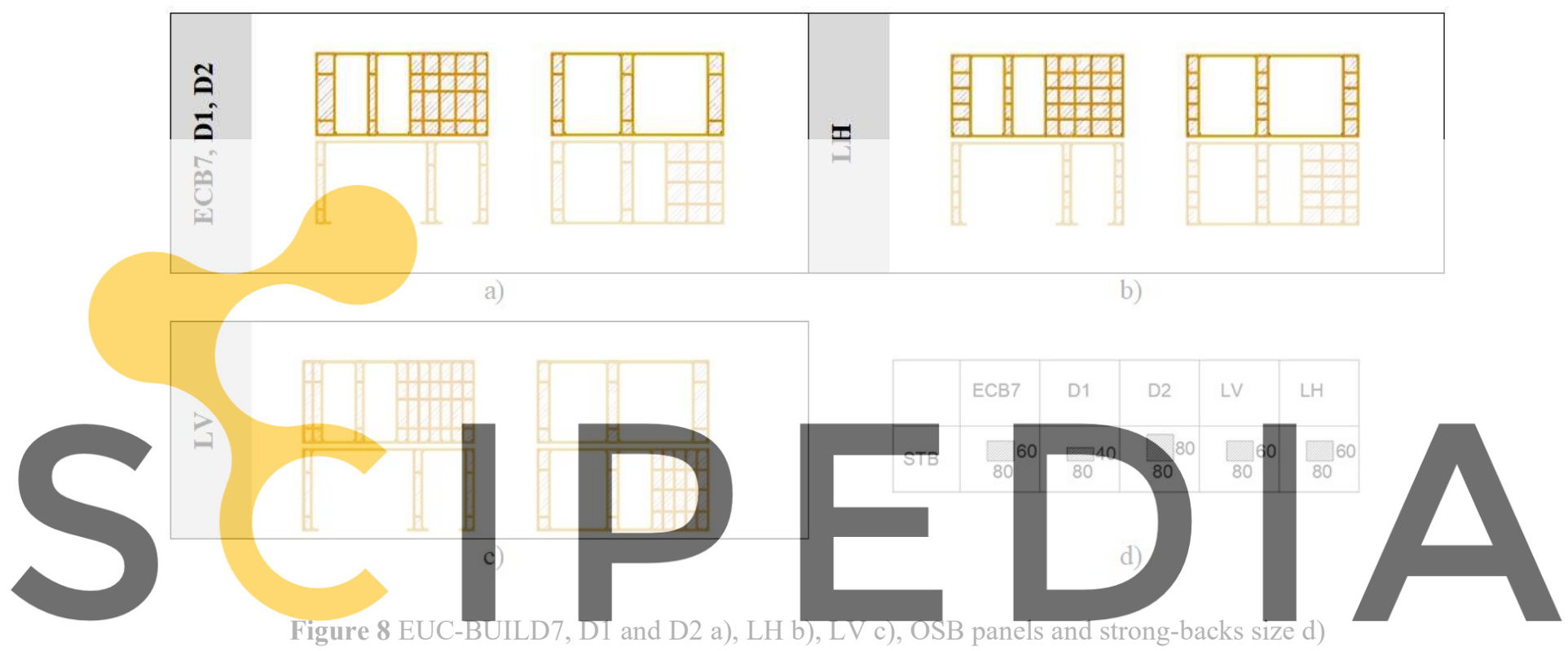

Register for free at https//www scipedia.com to download the version without the watermark section, a stiffer response, markedly lower displacements and higher values of lateral force capacity are obtained as in the case of configurations $\bar{L} \bar{H}, \mathrm{LV}$ and $\mathrm{D} 2$. When reducing the crosssection of the strong-backs instead (i.e. configuration D1), a more deformable solution is predicted (Figure 9). Since one of the objectives of the light retrofitting system presented by Miglietta et al. [5] is to propose a non-invasive retrofitting solution, based on these preliminary findings, a variation on the original layout can be introduced to improve further the building performance. Such a variation would involve reducing the horizontal or vertical timber frame span without compromising the slight footprint. Amongst the considered layout solutions, LH and LV would be preferable, since configuration D2 increases the retrofit footprint, where configuration D1 leads to a more deformable response. 

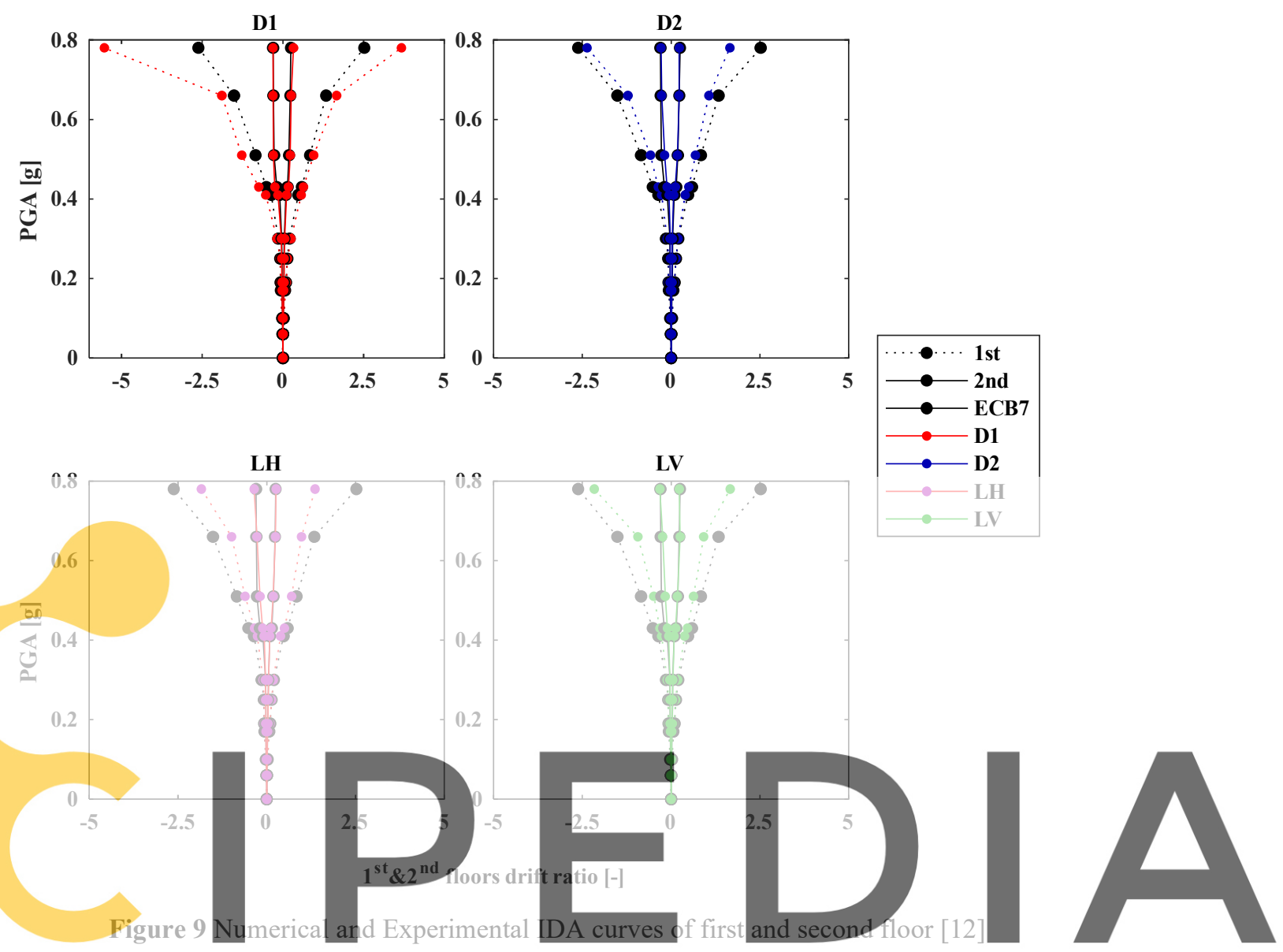

\section{Register for free at https $/$ Wwww.scipedia.com to download the version without the watermark}

The Groningen region, historically not prone to earthquakes, is recently affected by induced seismicity. This led to the development of several research programs aiming to mitigate the vulnerability of existing buildings. Amongst others the retrofitting system recently-tested in a shake-table test on a full-scale prototype at EUCENTRE (Pavia), in the framework of NAM project, seems to indicate that the dynamic response of the terraced house might be improved through the employment of such a reversible, non-invasive and cost-effective light retrofitting solution. In this work, the Applied Element Method is used to reproduce the dynamic response of the retrofitted building and to investigate numerically the influence of different geometrical configurations on the structural behaviour to optimise the original timber retrofit layout, since the goal of the light retrofitting system was to introduce a non-invasive solution. Each element of the retrofitting system is explicitly represented in the AEM model to reproduce the actual interaction with masonry walls. The numerical modelling strategy is firstly validated through comparison with experimental full-scale component tests on both non-retrofitted and retrofitted URM panels subjected to shear-compression cyclic loading protocol. The calibrated mechanical parameters are then implemented in the full-scale building model tested under the experimentally employed loading protocol. Considering the good agreement found in terms of 
displacements, lateral force capacity and damage, the validated building model is employed in a comprehensive parametric study to investigate the effectiveness of various retrofit layouts. To this end, a set of different configurations, designed to improve the structural performance without affecting the retrofit footprint, are numerically tested. Preliminary results of the parametric study presented in this work suggest that to optimise the retrofit efficacy, it would be preferable to vary horizontal or vertical timber elements span than to modify the elements frame dimensions, since a bigger section affects the retrofit footprint while a smaller one leads to a larger displacement. The AEM demonstrated to adequately reproduce the dynamic response of the original full-scale components and building specimen, being able to investigate the structural behaviour and being also a useful tool for the optimisation of the retrofit layout with the aim of finding a good solution to combine efficacy and minimum footprint.

\section{Acknowledgements.}

The work described in this paper was carried out within the framework of the research program on hazard and risk of induced seismicity in the Groningen region, sponsored by the Nederlandse Aardolie Maatschappij BV (NAM). The authors acknowledge all those at the European Centre for Training and Research in Earthquake Engineering (EUCENTRE, Pavia, Italy) that were involved in the testing campaign referred to in this paper, and in particular Nicolò Damiani, Marco Miglietta, Luca Grottoli, Gabriele Guerrini and Francesco Graziotti, for their precious assistance in accessing the test data. The collaboration of the technical support

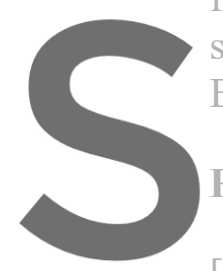
staff from Applied Science International LC (A
Extreme Loading for Structures, is also acknowl
REFERENCES

Meguro, K. and Tagel-Din, H. Applied element method for structural analysis: Theory and application for linear materials. Struct. Eng. Eartho. Eng. (2000) 1.7:21s-35s.

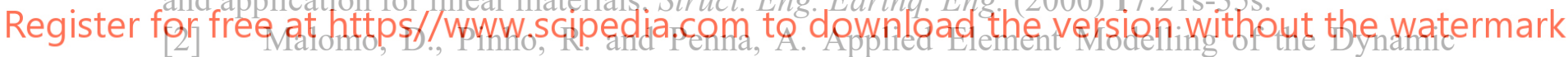
Response of a Full-Scale Clay Brick Masonry Building Specimen with Flexible Diaphragms.

Int. J. Archit. Herit. (2019) https://doi.org/10.1080/15583058.2019.1616004.

[3] Karbassi, A. and Nollet, M.J. Performance-based seismic vulnerability evaluation of masonry buildings using applied element method in a nonlinear dynamic-based analytical procedure. Earthq. Spectra (2013) 29:399-426.

[4] Van Elk, J.F., Bourne, S.J., Oates, S.J., Bommer, J., Pinho, R. and Crowley, H. A probabilistic model to evaluate options for mitigating induced seismic risk. Earthq. Spectra (2019) 35:537-64.

[5] Miglietta, M., Damiani, N., Guerrini, G. and Graziotti, F. Full-scale shake-table tests on two full-scale unreinforced masonry cavity-wall buildings: effect of an innovative timber retrofit system. Bull. Earthq. Eng. (to be submitted 2020).

[6] Graziotti, F., Penna, A. and Magenes G. A comprehensive in situ and laboratory testing programme supporting seismic risk analysis of URM buildings subjected to induced earthquakes. Bull. Earthq. Eng. (2019) 17:4575-99.

[7] Damiani, N., Miglietta, M., Mazzella, L., Grottoli, L., Guerrini, G. and Graziotti, F. Full-scale shaking table test on a Dutch URM cavity-wall terraced-house end unit - A retrofit 
solution with strong-backs and OSB boards - EUC-BUILD-7. EUCENTRE Foundation, Pavia, Italy, Research report EUC052/2019U (2019).

[8] Malomo, D., Pinho, R. and Penna, A. Using the applied element method for modelling calcium silicate brick masonry subjected to in-plane cyclic loading. Earthq. Eng. Struct. Dyn. (2018) 47:1610-30.

[9] Malomo, D., Comini, P., Pinho, R. and Penna A. The Applied Element Method and the modelling of both in-plane and out-of-plane response of URM walls. 16th Eur. Conf. Earthq. Eng., Thessaloniki, Greece (2018).

[10] Malomo, D., Morandini, C., Penna, A., Crowley, H. and Pinho, R. Impact of ground floor openings percentage on the dynamic response of URM cavity-wall structures. Bull. Earthq. Eng. (to be submitted 2020)

[11] Ravenshorst, G.J.P. and Mirra, M. Test report on cyclic behaviour of replicated timber diaphragms representing a detached house. Delft University of Technology, Delft, The Netherlands. Report number C31B67WP4-7, version 1, 30 December 2017 (2017).

[12] Morandini, C., Malomo, D., Pinho, R. and Penna, A. Simulation of shake table test on full-scale masonry building with timber retrofit using the applied element method. Eng. Struct. (to be submitted 2020)

[13] Guerrini, G., Damiani, N., Miglietta, M. and Graziotti, F. Cyclic response of masonry retrofitted with timber frames and boards. Struct. Build. (In press 2020) DOI: 10.1680/jstbu. 19.00134 